This article may be downloaded for personal use only. Any other use requires prior permission of the author and AIP Publishing. This article appeared in Surface Science Spectra, 11(1), 112-118 and may be found at https://doi.org/10.1116/11.20040804.

\title{
Analysis of Shea Nut Shells by X-ray Photoelectron Spectroscopy
}

Guilin Jiang, Ghaleb A. Husseini, ${ }^{\text {a) }}$ Larry L. Baxter, and Matthew R. Linford

Brigham Young University, Provo, Utah 84604

(Received 12 August 2004; accepted 16 November 2005; published 30 December 2005)

Determining the chemical structure and composition of biomass fuels using x-ray photoelectron spectroscopy (XPS) can provide fundamental knowledge of their structures that is useful in understanding and predicting their combustion behavior. Shea nut shells are an example of an agricultural residue (byproduct of food and feed production) of potential interest for biomass combustion. The XPS spectra of shea nut shells provide both its elemental composition and indications of its bonding. Traditional fuel analyses of this fuel are also provided. These include: ultimate analysis - the elemental composition of the overall fuel $(\mathrm{C}, \mathrm{H}, \mathrm{N}, \mathrm{S}$, and $\mathrm{O})$; chlorine analysis - reported here as part of the ultimate analysis but formally a separate procedure; proximate analysis - the proximate composition of the fuel (moisture, fixed carbon, volatiles, and ash); heating value - the specific heat of combustion; ash chemistry analysis — an elemental analysis of the ash content, expressed as oxides (which does not imply that they occur as oxides in the fuel). These data are summarized with the XPS spectra. (c) 2005 American Vacuum Society. [DOI: $10.1116 / 11.20040804]$

Keywords: biomass; shea nut shells; XPS; fuel

PACS: $82.80 . P$ v, 01.30.Kj, 84.60.Rb, 82.33.Vx, 82.60.Cx

\section{SPECIMEN DESCRIPTION}

Host Material: shea nut shells

Host Material Characteristics: homogeneous; amorphous; unknown electrical characteristics; biological material; powder

Chemical Name: cellulose

Host Composition: see entry for History \& Significance

Form: powder

History \& Significance: Shea nut shells are an example of an agricultural residue (byproduct of food and feed production) of potential interest for biomass combustion. The XPS spectra of shea nut shells provide both its elemental composition and indications of its bonding. Traditional fuel analyses of this fuel are also provided. These include: ultimate analysis - the elemental composition of the overall fuel $(\mathrm{C}, \mathrm{H}, \mathrm{N}, \mathrm{S}$, and $\mathrm{O})$; chlorine analysis - reported here as part of the ultimate analysis but formally a separate procedure; proximate analysis the proximate composition of the fuel (moisture, fixed carbon, volatiles, and ash); heating value - the specific heat of combustion; ash chemistry analysis - an elemental analysis of the ash content, expressed as oxides (which does not imply that they occur as oxides in the fuel). These data are summarized with the XPS spectra. The chemical composition of Shea nut shells is summarized in Tables 1 and 2.

As Received Condition: powder

Analyzed Region: same as host material

Ex Situ Preparation/Mounting: Sawdust powders were used as received. The powders were pressed onto a piece of nonconductive double-sticky tape mounted on a piece of silicon,

a) Author to whom correspondence should be addressed; present address: Chemical Engineering Department, P.O. Box 26666, The American University of Sharjah, Sharjah, United Arab Emirates.

\author{
Accession \# 00898 \\ Technique: XPS \\ Host Material: shea nut shells \\ Instrument: Surface Science \\ Instruments SSX-100 \\ Major Elements in Spectrum: C, O \\ Minor Elements in Spectrum: $\mathrm{N}$ \\ Printed Spectra: 7 \\ Spectra in Electronic Record: 7 \\ Spectral Category: technical
}

\begin{tabular}{lc}
\hline \begin{tabular}{l} 
Table 1: Fuel analysis of bio-fuel sunflower shells \\
(ash free basis except for ash and LHV, which are \\
on an as-received basis). \\
\hline
\end{tabular} \\
\cline { 2 - 2 } Moisture & $\%$ by weight \\
C & 9.84 \\
H & 46.40 \\
O & 5.85 \\
N & 34.33 \\
S & 0.88 \\
Ash & 0.18 \\
Sum & 2.52 \\
LHV*, MJ/kg & 100.0 \\
\hline
\end{tabular}

*Lower heating value

Table 2: Ash composition of sunflower shells (percent of ash basis).

\begin{tabular}{lc}
\hline & Mass $\%$ \\
\cline { 2 - 2 } $\mathrm{SiO}_{2}$ & 1.1 \\
$\mathrm{Al}_{2} \mathrm{O}_{3}$ & 0.5 \\
$\mathrm{Fe}_{2} \mathrm{O}_{3}$ & 0.9 \\
$\mathrm{CaO}$ & 16 \\
$\mathrm{MgO}$ & 13.1 \\
$\mathrm{Na}_{2} \mathrm{O}$ & $<0.2$ \\
$\mathrm{~K}_{2} \mathrm{O}$ & 45.1 \\
$\mathrm{SO}_{3}$ & 11.7 \\
$\mathrm{P}_{2} \mathrm{O}_{5}$ & 10.1 \\
$\mathrm{Cl}$ & 1.2 \\
Other & 0.3 \\
Sum & 100 \\
\hline
\end{tabular}


which was then mounted on the sample stage with a piece of the same tape.

\section{In Situ Preparation: none}

Pre-Analysis Beam Exposure: No damage was observed in the sample even after several hours of exposure to X-ray radiation. After $4 \mathrm{~h}$ of exposure to $\mathrm{x}$ rays, the intensity of the $\mathrm{N} 1 s$ scan did not change.

Charge Control: A flood gun was applied. The flood gun voltage was $4 \mathrm{~V}$, and its current was less than $50 \mathrm{~mA}$. A metal screen was used to mask the sample. The charge control was determined by observing zirconia $\mathrm{Zr} 3 p_{3 / 2}$ peak positions under different flood gun settings. XPS spectra showed a $\mathrm{Zr} 3 d_{5 / 2}$ at $182.3 \mathrm{eV}$. The metal screen used was nickel, $1 \mathrm{~mm}$ distance, 70 lines/in. and $90 \%$ transmission.

Temp. During Analysis: $298 \mathrm{~K}$

Pressure During Analysis: $<2.0 \times 10^{-6} \mathrm{~Pa}$

INSTRUMENT DESCRIPTION

Manufacturer and Model: Surface Science Instruments SSX-100 Analyzer Type: spherical sector

Detector: resistive anode position detector

Number of Detector Elements: 128

INSTRUMENT PARAMETERS COMMON TO ALL SPECTRA

\section{Spectrometer}

Analyzer Mode: constant pass energy

Throughput $\left(\boldsymbol{T}=\boldsymbol{E}^{N}\right): N=0$
Excitation Source Window: $12 \mu \mathrm{m}$ aluminum foil

Excitation Source: $\mathrm{Al} K_{\alpha}$ monochromatic

Source Energy: $1486.6 \mathrm{eV}$

Source Strength: $200 \mathrm{~W}$

Signal Mode: multichannel direct

Geometry

Incident Angle: $55^{\circ}$

Source to Analyzer Angle: $70.8^{\circ}$

Emission Angle: $55^{\circ}$

Specimen Azimuthal Angle: $0^{\circ}$

Acceptance Angle from Analyzer Axis: $0^{\circ}$

\section{DATA ANALYSIS METHOD}

Peak Shape and Background Method: Shirley background function

Quantitation Method: Sensitivity factors were obtained from ESCA 2000 NT software supplied by Service Physics. The peak areas are the areas above a linear background.

\section{ACKNOWLEDGMENTS}

The authors acknowledge U.S. DOE Biomass Power Program for financial support, and Elsam engineering and Eltra, both Danish companies, which provided complementary analyses and some financial support for this investigation. 
SPECTRAL FEATURES TABLE

\begin{tabular}{|c|c|c|c|c|c|c|c|}
\hline $\begin{array}{l}\text { Spectrum } \\
\text { ID \# }\end{array}$ & $\begin{array}{l}\text { Element/ } \\
\text { Transition }\end{array}$ & $\begin{array}{c}\text { Peak } \\
\text { Energy } \\
(\mathrm{eV})\end{array}$ & $\begin{array}{c}\text { Peak Width } \\
\text { FWHM } \\
(\mathrm{eV})\end{array}$ & $\begin{array}{c}\text { Peak Area } \\
\text { (counts) }\end{array}$ & $\begin{array}{c}\text { Sensitivity } \\
\text { Factor }\end{array}$ & $\begin{array}{l}\text { Concen- } \\
\text { tration } \\
\text { (at. \%) }\end{array}$ & $\begin{array}{c}\text { Peak } \\
\text { Assignment }\end{array}$ \\
\hline 00898-02 & O $1 s$ & 528.6 & 3.3 & 67700 & 2.5 & 20 & $\ldots$ \\
\hline 00898-03 & $\mathrm{C} 1 s$ & 281.4 & 3.8 & 106000 & 1 & 78.2 & $\ldots$ \\
\hline 00898-04 & $\mathrm{N} 1 s$ & 396.2 & 4.8 & 39700 & 1.68 & 1.7 & $\cdots$ \\
\hline 00898-05 & $\mathrm{O} 1 s$ & 529.0 & 3.1 & 26400 & 2.5 & $\ldots$ & $\ldots$ \\
\hline 00898-06 & C $1 s$ & 281.9 & 3.2 & 37000 & 1 & $\ldots$ & $\ldots$ \\
\hline 00898-07 & $\mathrm{N} 1 s$ & 396.7 & 3.5 & 50000 & 1.68 & $\cdots$ & $\cdots$ \\
\hline
\end{tabular}

\begin{tabular}{|c|c|c|c|c|c|}
\hline \multicolumn{6}{|c|}{ GUIDE TO FIGURES } \\
\hline $\begin{array}{l}\text { Spectrum } \\
\text { (Accession) \# }\end{array}$ & $\begin{array}{l}\text { Spectral } \\
\text { Region }\end{array}$ & $\begin{array}{l}\text { Voltage } \\
\text { Shift* }^{\star}\end{array}$ & Multiplier & Baseline & Comment \# \\
\hline 898-1 & Survey & 0 & 1 & 0 & 1 \\
\hline $898-2$ & $\mathrm{O} 1 s$ & 0 & 1 & 0 & 1 \\
\hline $898-3$ & $\mathrm{C} 1 s$ & 0 & 1 & 0 & 1 \\
\hline $898-4$ & $\mathrm{~N} 1 s$ & 0 & 1 & 0 & 1 \\
\hline $898-5$ & $\mathrm{O} 1 s$ & 0 & 1 & 0 & 2 \\
\hline 898-6 & C $1 s$ & 0 & 1 & 0 & 2 \\
\hline 898-7 & $\mathrm{N} 1 s$ & 0 & 1 & 0 & 2 \\
\hline
\end{tabular}

* Voltage shift of the archived (as-measured) spectrum relative to the printed figure. The figure reflects the recommended energy scale correction due to a calibration correction, sample charging, flood gun, or other phenomenon.

1. $800 \mu \mathrm{m}$ x-ray beam diameter, $150 \mathrm{eV}$ pass energy

2. $300 \mu \mathrm{m} x$-ray beam diameter, $50 \mathrm{eV}$ pass energy 


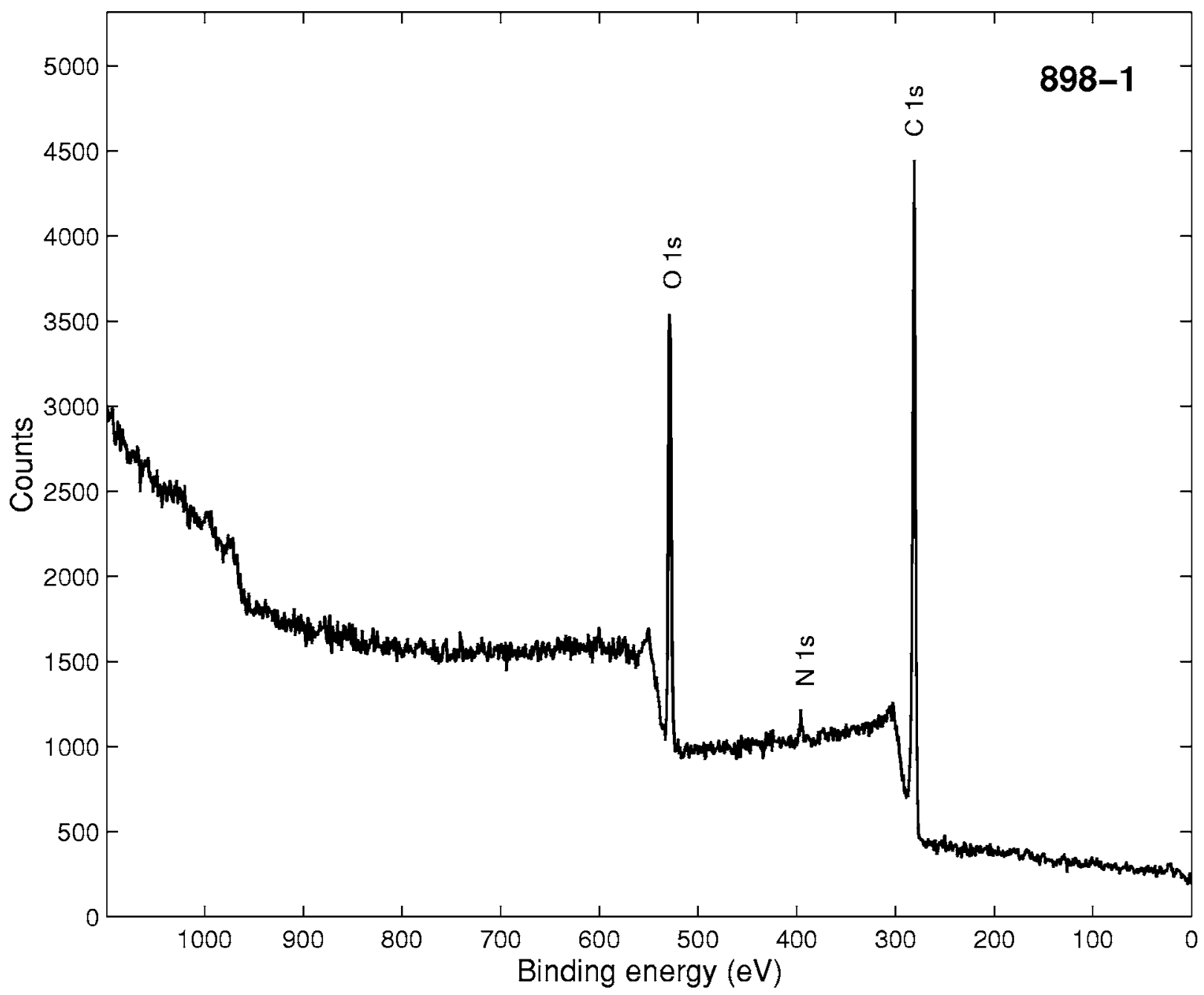

\begin{tabular}{|c|c|}
\hline Accession \# & 00898-01 \\
\hline Host Material & shea nut shells \\
\hline Technique & XPS \\
\hline Spectral Region & survey \\
\hline Instrument & Surface Science Instruments SSX-100 \\
\hline Excitation Source & Al $K_{\alpha}$ monochromatic \\
\hline Source Energy & $1486.6 \mathrm{eV}$ \\
\hline Source Strength & $200 \mathrm{~W}$ \\
\hline Source Size & $0.8 \mathrm{~mm} \times 0.8 \mathrm{~mm}$ \\
\hline Analyzer Type & spherical sector \\
\hline Incident Angle & $55^{\circ}$ \\
\hline Emission Angle & $55^{\circ}$ \\
\hline Analyzer Pass Energy & $150 \mathrm{eV}$ \\
\hline Analyzer Resolution & $1.5 \mathrm{eV}$ \\
\hline Total Signal Accumulation Time & $2200 \mathrm{~s}$ \\
\hline Total Elapsed Time & $2400 \mathrm{~s}$ \\
\hline Number of Scans & 10 \\
\hline Source Beam Size at Specimen Surface & $0.8 \mathrm{~mm} \times 1.392 \mathrm{~mm}$ \\
\hline Effective Detector Width & $19 \mathrm{eV}$ \\
\hline Analyzer Width & $1500 \mu \mathrm{m} \times 12000 \mu \mathrm{m}$ at $84 \mathrm{eV}$ \\
\hline
\end{tabular}






- Accession \#: 00898-02

- Host Material: shea nut shells

- Technique: XPS

- Spectral Region: $01 \mathrm{~s}$

Instrument: Surface Science Instruments SSX-100

Excitation Source: $\mathrm{Al} K_{\alpha}$ monochromatic

Source Energy: $1486.6 \mathrm{eV}$ Source Strength: $200 \mathrm{~W}$

Source Size: $0.8 \mathrm{~mm} \times 0.8 \mathrm{~mm}$ Incident Angle: $55^{\circ}$

Analyzer Type: spherical sector Analyzer Pass Energy: $150 \mathrm{eV}$ Analyzer Resolution: $1.5 \mathrm{eV}$

Emission Angle: $55^{\circ}$

Total Signal Accumulation Time: $306.5 \mathrm{~s}$

Total Elapsed Time: $475.5 \mathrm{~s}$

Number of Scans: 5

Source Beam Size at Specimen Surface: $0.8 \mathrm{~mm} \times 1.392 \mathrm{~mm}$ Effective Detector Width: $19 \mathrm{eV}$ Analyzer Width: $1500 \mu \mathrm{m} \times$ $12000 \mu \mathrm{m}$ at $84 \mathrm{eV}$

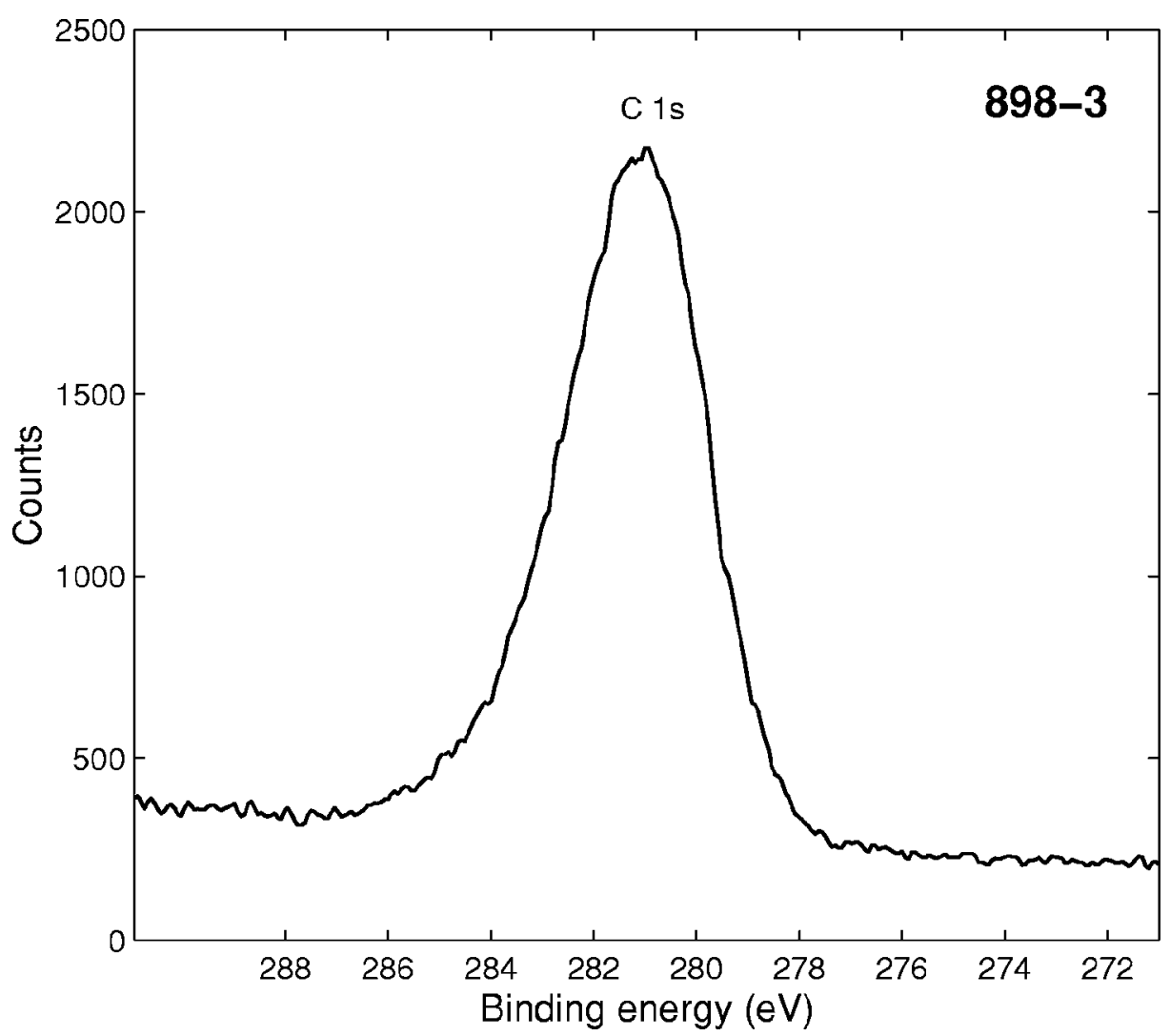

- Accession \#: 00898-03

- Host Material: shea nut shells

- Technique: XPS

- Spectral Region: C $1 \mathrm{~s}$

Instrument: Surface Science Instruments SSX-100

Excitation Source: Al $K_{\alpha}$ monochromatic

Source Energy: $1486.6 \mathrm{eV}$

Source Strength: $200 \mathrm{~W}$

Source Size: $0.8 \mathrm{~mm} \times 0.8 \mathrm{~mm}$ Incident Angle: $55^{\circ}$

Analyzer Type: spherical sector Analyzer Pass Energy: $150 \mathrm{eV}$ Analyzer Resolution: $1.5 \mathrm{eV}$ Emission Angle: $55^{\circ}$

Total Signal Accumulation Time: $306.5 \mathrm{~s}$

Total Elapsed Time: $475.5 \mathrm{~s}$

Number of Scans: 5

Source Beam Size at Specimen Surface: $0.8 \mathrm{~mm} \times 1.392 \mathrm{~mm}$ Effective Detector Width: $19 \mathrm{eV}$ Analyzer Width: $1500 \mu \mathrm{m} \times$ $12000 \mu \mathrm{m}$ at $84 \mathrm{eV}$ 


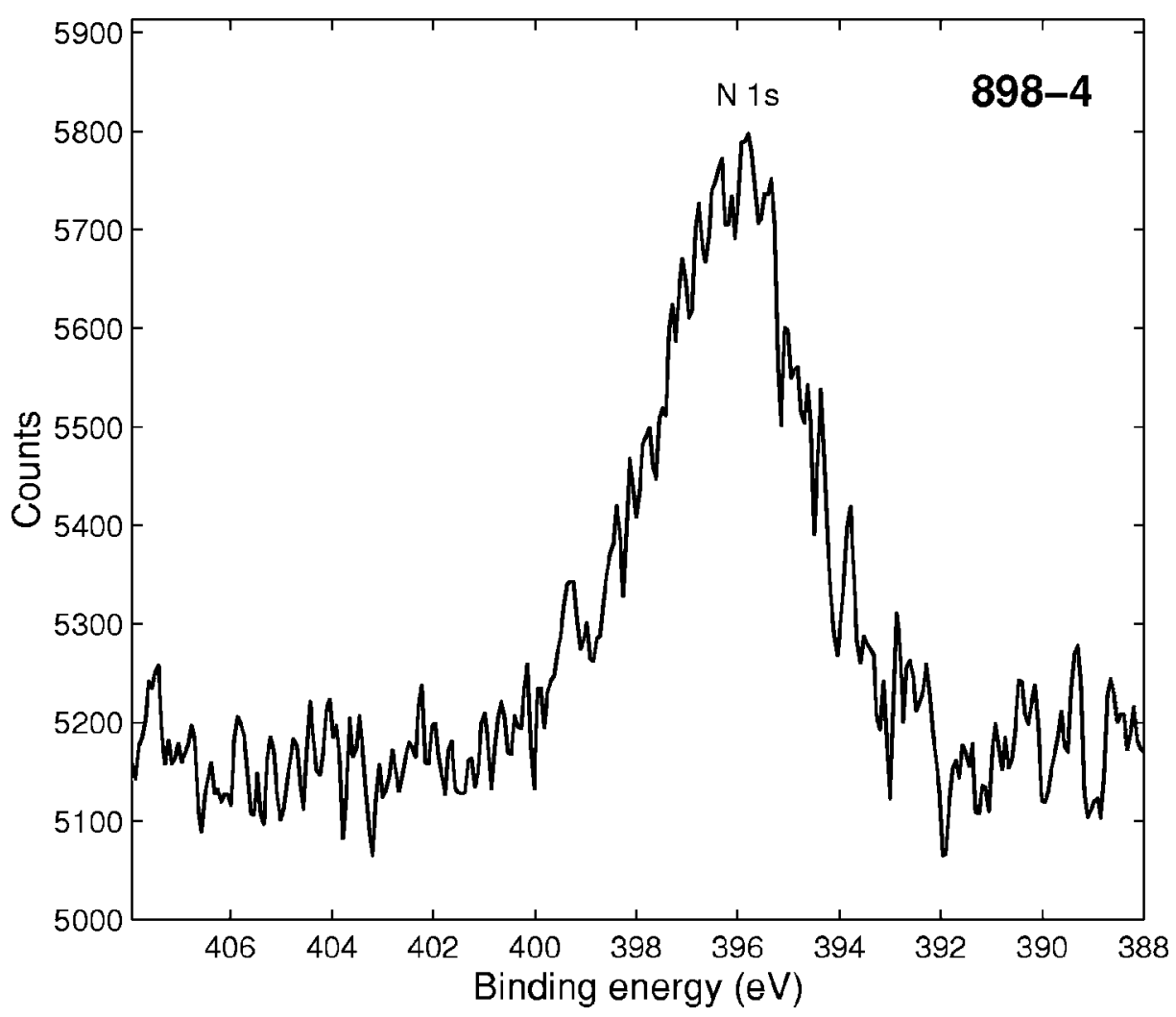

- Accession \#: 00898-04

- Host Material: shea nut shells

- Technique: XPS

- Spectral Region: N1s

Instrument: Surface Science Instruments SSX-100

Excitation Source: Al $K_{\alpha}$ monochromatic

Source Energy: $1486.6 \mathrm{eV}$

Source Strength: $200 \mathrm{~W}$

Source Size: $0.8 \mathrm{~mm} \times 0.8 \mathrm{~mm}$ Incident Angle: $55^{\circ}$

Analyzer Type: spherical sector Analyzer Pass Energy: $150 \mathrm{eV}$

Analyzer Resolution: $1.5 \mathrm{eV}$

Emission Angle: $55^{\circ}$

Total Signal Accumulation Time: $3065 \mathrm{~s}$

Total Elapsed Time: $3234 \mathrm{~s}$

Number of Scans: 50

Source Beam Size at Specimen Surface: $0.8 \mathrm{~mm} \times 1.392 \mathrm{~mm}$ Effective Detector Width: $19 \mathrm{eV}$ Analyzer Width: $1500 \mu \mathrm{m} \times$ $12000 \mu \mathrm{m}$ at $84 \mathrm{eV}$

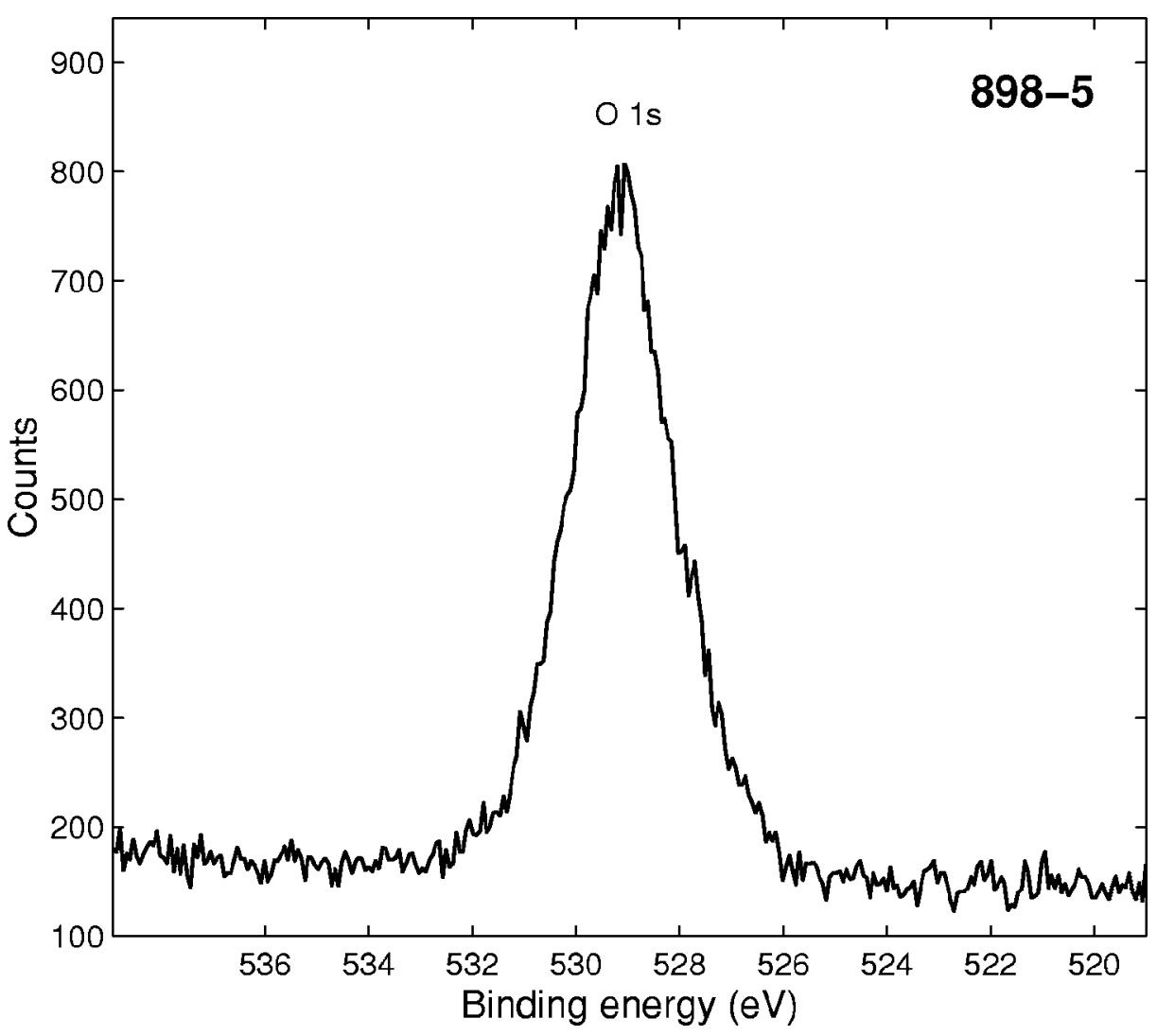

- Accession \#: 00898-05

- Host Material: shea nut shells

- Technique: XPS

- Spectral Region: $01 \mathrm{~s}$

Instrument: Surface Science Instruments SSX-100

Excitation Source: Al $K_{\alpha}$ monochromatic

Source Energy: $1486.6 \mathrm{eV}$

Source Strength: $200 \mathrm{~W}$

Source Size: $0.3 \mathrm{~mm} \times 0.3 \mathrm{~mm}$ Incident Angle: $55^{\circ}$

Analyzer Type: spherical sector Analyzer Pass Energy: $50 \mathrm{eV}$ Analyzer Resolution: $0.5 \mathrm{eV}$

Emission Angle: $55^{\circ}$

Total Signal Accumulation Time: $613 \mathrm{~s}$

Total Elapsed Time: $782 \mathrm{~s}$

Number of Scans: 10

Source Beam Size at Specimen Surface: $0.3 \mathrm{~mm} \times 0.523 \mathrm{~mm}$ Effective Detector Width: $6.6 \mathrm{eV}$ Analyzer Width: $750 \mu \mathrm{m} \times$ $6000 \mu \mathrm{m}$ at $84 \mathrm{eV}$ 


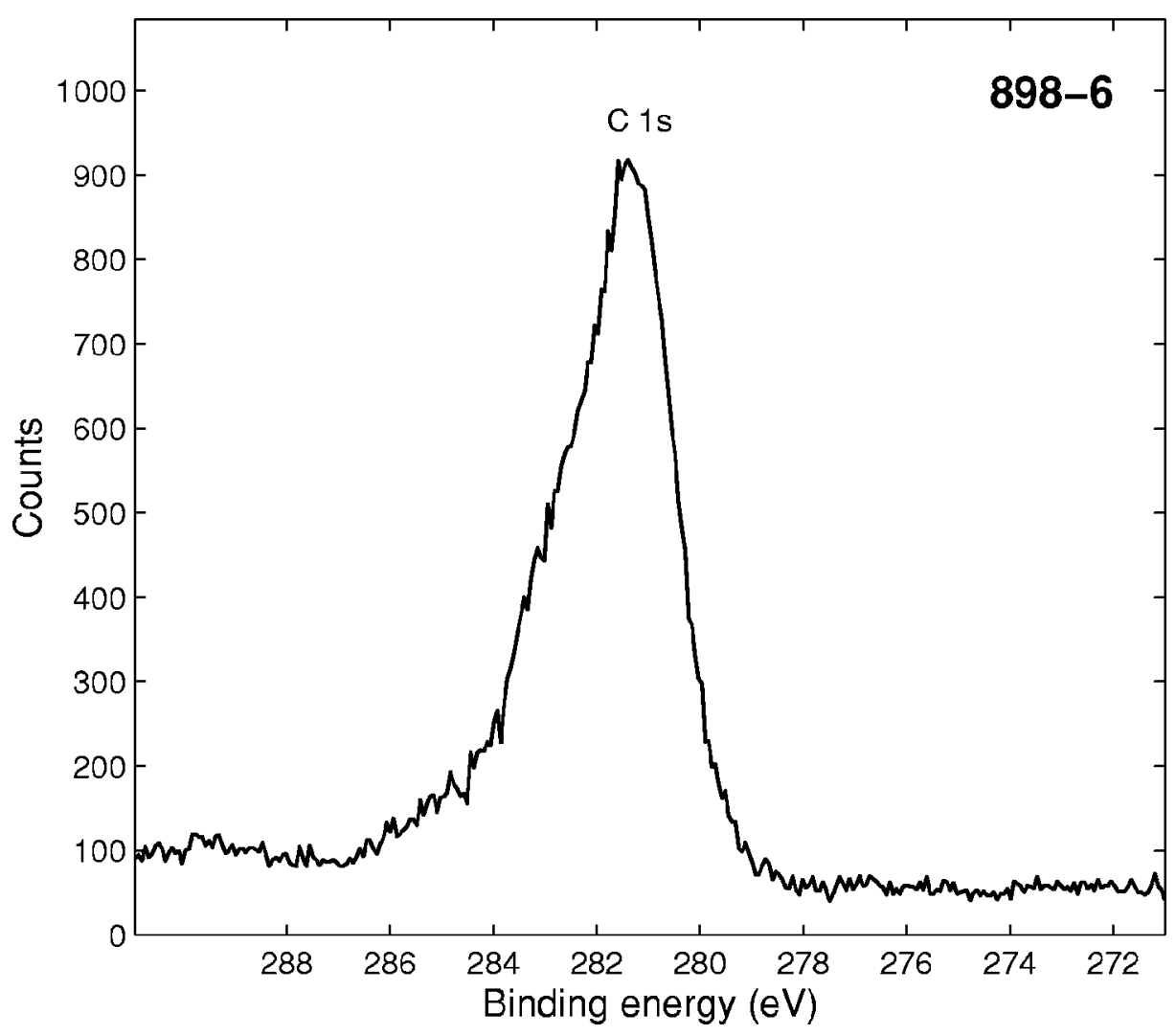

- Accession \#: 00898-06

- Host Material: shea nut shells

- Technique: XPS

- Spectral Region: C $1 s$

Instrument: Surface Science Instruments SSX-100

Excitation Source: Al $K_{\alpha}$ monochromatic

Source Energy: $1486.6 \mathrm{eV}$

Source Strength: $200 \mathrm{~W}$

Source Size: $0.3 \mathrm{~mm} \times 0.3 \mathrm{~mm}$ Incident Angle: $55^{\circ}$

Analyzer Type: spherical sector Analyzer Pass Energy: $50 \mathrm{eV}$

Analyzer Resolution: $0.5 \mathrm{eV}$

Emission Angle: $55^{\circ}$

Total Signal Accumulation Time: $613 \mathrm{~s}$

Total Elapsed Time: $782 \mathrm{~s}$

Number of Scans: 10

Source Beam Size at Specimen Surface: $0.3 \mathrm{~mm} \times 0.523 \mathrm{~mm}$ Effective Detector Width: $6.6 \mathrm{eV}$ Analyzer Width: $750 \mu \mathrm{m} \times$ $6000 \mu \mathrm{m}$ at $84 \mathrm{eV}$



- Accession \#: 00898-07

- Host Material: shea nut shells

- Technique: XPS

- Spectral Region: N1s

Instrument: Surface Science Instruments SSX-100

Excitation Source: Al $K_{\alpha}$ monochromatic

Source Energy: $1486.6 \mathrm{eV}$

Source Strength: $200 \mathrm{~W}$

Source Size: $0.3 \mathrm{~mm} \times 0.3 \mathrm{~mm}$ Incident Angle: $55^{\circ}$

Analyzer Type: spherical sector Analyzer Pass Energy: $50 \mathrm{eV}$ Analyzer Resolution: $0.5 \mathrm{eV}$

Emission Angle: $55^{\circ}$

Total Signal Accumulation Time: $24520 \mathrm{~s}$

Total Elapsed Time: $24689 \mathrm{~s}$

Number of Scans: 400

Source Beam Size at Specimen Surface: $0.3 \mathrm{~mm} \times 0.523 \mathrm{~mm}$ Effective Detector Width: $6.6 \mathrm{eV}$ Analyzer Width: $750 \mu \mathrm{m} \times$ $6000 \mu \mathrm{m}$ at $84 \mathrm{eV}$ 